\title{
The information simplicity of scoring rules
}

Citation for published version (APA):

Nuñez, M., \& Valletta, G. (2012). The information simplicity of scoring rules. METEOR, Maastricht University School of Business and Economics. METEOR Research Memorandum No. 011 https://doi.org/10.26481/umamet.2012011

Document status and date:

Published: 01/01/2012

DOI:

10.26481/umamet.2012011

Document Version:

Publisher's PDF, also known as Version of record

\section{Please check the document version of this publication:}

- A submitted manuscript is the version of the article upon submission and before peer-review. There can be important differences between the submitted version and the official published version of record.

People interested in the research are advised to contact the author for the final version of the publication, or visit the DOI to the publisher's website.

- The final author version and the galley proof are versions of the publication after peer review.

- The final published version features the final layout of the paper including the volume, issue and page numbers.

Link to publication

\footnotetext{
General rights rights.

- You may freely distribute the URL identifying the publication in the public portal. please follow below link for the End User Agreement:

www.umlib.nl/taverne-license

Take down policy

If you believe that this document breaches copyright please contact us at:

repository@maastrichtuniversity.nl

providing details and we will investigate your claim.
}

Copyright and moral rights for the publications made accessible in the public portal are retained by the authors and/or other copyright owners and it is a condition of accessing publications that users recognise and abide by the legal requirements associated with these

- Users may download and print one copy of any publication from the public portal for the purpose of private study or research.

- You may not further distribute the material or use it for any profit-making activity or commercial gain

If the publication is distributed under the terms of Article $25 \mathrm{fa}$ of the Dutch Copyright Act, indicated by the "Taverne" license above, 


\section{Maastricht University}

Matías Núñez, Giacomo Valleta

The information simplicity of scoring rules

$\mathrm{RM} / 12 / 011$

\section{METEOR}

Maastricht University School of Business and Economics

Maastricht Research School of Economics

of Technology and Organization

P. B. Box 616

NL -6200 MD Maastricht

The Netherlands 


\title{
The Informational Simplicity of Scoring Rules.*
}

\author{
Matías Núñez ${ }^{\dagger}$ and Giacomo Valletta ${ }^{\ddagger}$
}

February 2012

\begin{abstract}
Voters assign a score to each of the many available alternatives. We study the normative properties of procedures that aggregate the scores collected in the ballot box. A vast class of aggregation procedures, including procedures based on the pairwise comparison of alternatives, satisfy May's famous conditions in our framework. We prove that, within such a plethora of procedures, scoring rules are singled out by a property related to their informational simplicity: in order to determine the winner, they do not take into account the specific distribution of scores chosen by each voter. The result is shown to hold regardless of the introduction of asymmetry among the alternatives.
\end{abstract}

KEYWORDS: Scoring Rules, May's Theorem, Informational Simplicity.

The design of voting procedures has attracted a considerable interest both in positive political theory and social choice theory. The simplest situation one could possibly conceive is the choice among two alternatives. In such a binary setting, the fairest solution seems to be majority voting as this is the only voting procedure that satisfies the compelling conditions proposed by May (1952) [23]. Majority voting is a well defined voting procedure for any profile of announcements over the two alternatives (Universal Domain), it treats the voters (Anonymity) and the alternatives (Neutrality) symmetrically and if some voters change their vote in favor of one alternative the outcome of the election changes accordingly (Positive Responsiveness).

If the choice among three or more alternatives is at stake, things become considerably more complicated. Still, the most natural solution seems to be plurality voting, namely, given the preference ordering over the alternatives of each voter, choose the alternative that is ranked first by the largest number of them. However,

\footnotetext{
${ }^{*}$ This work has benefited from useful discussions with Ani Guerdjikova, Sébastien Courtin, Jean-François Laslier, François Maniquet, Mathieu Martin, Vincent Merlin, Marcus Pivato, Kaj Thomsson and seminar participants at CORE.

${ }^{\dagger}$ CNRS and Université de Cergy-Pontoise, THEMA, F-95000 Cergy-Pontoise, France. Email: matias.nunez@u-cergy.fr.

${ }^{\ddagger}$ Maastricht University, Netherlands - Email: g.valletta@maastrichtuniversity.nl.
} 
as it is well-known, this rule has a number of serious drawbacks. For instance, it can lead to the choice of an alternative that ranks very low in the preferences of the voters as early noticed both by Condorcet and Borda. They proposed two distinct solutions that were meant to (partially) solve this problem. The former suggested that the alternative defeating every other alternative in pairwise comparisons (the Condorcet Winner), if it exists, should be the winner of the election. The latter proposed the Borda count: each alternative receives a score which equals its ranking in the ordering and the winner of the election is the alternative who collects the highest total score. These two ideas provide the underpinnings for the two most extensively studied families of voting procedures: Condorcet procedures (based on the pairwise comparisons of alternatives) and scoring rules. Scoring rules are simple and widely used voting procedures. A voter assigns a certain number of points to each of the alternatives and those which obtain the highest total score (i.e. the sum of points) are the winners of the election ${ }^{1}$.

In order to merely formulate the aforementioned weakness of plurality voting (and to fix it), one has to refer to voters' preference orderings. Indeed most of the literature in social choice theory assumes, implicitly or explicitly, that when voters cast their ballot, they have to report their preference ordering over the available alternatives. $^{2}$ This need not be the case in real-life elections. The amount of information asked to each voter depends on the voting procedure. For example, under plurality voting, voters are asked to reveal their "first choice" whereas under the Borda rule they have to provide much more information. Moreover, even if two voting procedures ask to the voters the same information they do not necessarily use the same amount of it in order to determine the winner. Indeed, both the Borda Rule and the Condorcet Rule collect the same amount of information from the voters but they use a different amount of it: the former just considers the total score received by each alternative and the latter hinges, for each couple of alternative, on the relative score assigned by each voter.

In order to better understand the informational basis of voting procedures ${ }^{3}$ one

\footnotetext{
${ }^{1}$ Scoring rules differ according to which range of points voters are allowed to assign to different alternatives. For instance, plurality and approval voting (in which a voter can assign one point to as many alternatives as she wishes) are both scoring rules.

${ }^{2}$ One has to refer to voters' preference orderings. Indeed most of the literature in social choice theory assumes, implicitly or explicitly, that when voters cast their ballot, they have to report their preference ordering over the available alternatives. This is in fact behind the plethora of negative results we can find in such a literature. Arrow's (1951) [2] General Possibility theorem and the Gibbard-Satterthwaite theorem on strategic manipulability (Gibbard (1973) [14]; Satterthwaite (1975) [30]) stand among the most influential results. A recent stream of the literature focuses on the desirability of manipulability of voting procedures (see for example Dowding and van Hees (2008) [11]). Again, the basic input of such studies is the voters' preference orderings. Considerations about the manipulability of a voting procedure are beyond the scope of our paper.

${ }^{3}$ The literature on the informational basis of the measurement of individual welfare and their relation with the measure of collective welfare, the so-called informational basis of social choice (Sen (1970) [32], d'Aspremont and Gevers (1977) [10] and Fleurbaey (1993) [13]), is very large.
} 
could think of them as consisting of two parts. The first one defines the amount of information one should ask to the voters, the balloting problem. The second one, determines how to use such an information in order to designate the winner(s), the aggregation problem ${ }^{4}$. The objective of the balloting problem is to devise the ideal shape of the balloting procedure of an election. The aim of the aggregation problem ${ }^{5}$, is to single out a desirable aggregation procedure given the information available.

Among the properties that define the desirability of an aggregation procedure, one should consider its informational simplicity. That is, the difference between the amount of information deriving from the balloting procedure and the amount actually used by the aggregation procedure. For instance, as explained earlier, the Borda and the Condorcet rule differ from the informational simplicity point of view. We provide a characterization of the entire family of scoring rules based on their informational simplicity. Within a vast family of voting procedures scoring rules are the only ones that look at the information contained in the ballot box in its entirety neglecting the fact that a score assigned to an alternative belongs to a specific distribution of scores chosen by each voter.

In order to make as clear as possible the distinction between the amount of information collected by a voting procedure and amount actually used we only focus on the normative properties of the aggregation procedure, taking as given the balloting procedure. To the best of our knowledge the only paper that follows the same route is Goodin and List (2006) [17]. The authors provide an interesting generalization of May's theorem to many-option decisions provided that the balloting procedure collects only one vote (her revealed first-choice) from each voter (instead of asking her the full preference ordering over the alternatives). Their work stands out as a "conditional defense" of plurality voting, conditional to the limited amount of information asked to the voters by the balloting procedure.

We follow a similar line of reasoning but we move a step further by considering a wider family of balloting procedures. We do so by allowing each voter to cast several ballots, each ballot standing for one alternative. The amount of ballots that

Surprisingly there is not a counterpart of such a literature in voting theory.

${ }^{4}$ Such a distinction is seldom made in the literature. To the best of our knowledge, one of the first works which emphasized the importance of such a distinction is Merril and Nagel (1987) [25] where it is argued that "in the recent literature, the conflation of balloting method and decision rule through the phrase "plurality method" has become a source of confusion not just in language but also in thinking."

${ }^{5}$ One should notice that different aggregation procedures can be associated to the same balloting procedure. For example full-preference balloting can be used by assuming that the winner of the election is the alternative with the highest sum of votes or the one with at least $2 / 3$ of the votes. On the contrary, the same aggregation procedure can be based on different balloting procedure. For example, declaring as winner(s) the alternative(s) with the most votes can be defined on single vote balloting (and this is typically happens in real world situations where voters are asked to vote for one alternative only) or full-preference balloting (for instance Borda balloting). 
each voter can cast for each alternative depends on the balloting procedure chosen ${ }^{6}$. Practically speaking, each voter can assign a score to each of the alternatives (each score being a positive integer). This particular way of representing balloting procedures is fully general and it just simplifies the definition the normative properties bearing on the aggregation procedures.

We start our analysis focusing on the conditions proposed by May. To do so, we adapt them to our framework, a score-based informational environment with many alternatives, keeping unaltered their normative appeal. Not surprisingly, in such a framework, these conditions are not particularly restrictive: many aggregation procedures satisfy them. For example, both procedures based on the sum of scores, like approval voting and the Borda rule, and Condorcet procedures, such as the Black or the Copeland procedure, satisfy these conditions. But if we strengthen Anonymity in such a way that the information about the combination of ballots cast by a voter (and not only her identity) is not relevant for the aggregation procedure then we are only left with aggregation procedures based on the sum of the scores, the scoring rules. That is, choosing the option(s) that has collected the highest number of ballots in the ballot box is the only desirable aggregation procedure.

Our result has two interpretations of particular interest.

First, we provide a characterization of scoring rules that relies on a condition that describes their informational simplicity. This condition is behind a property often used in strategic voting theory as it greatly simplifies the analysis of the electoral game: the specific composition of the message sent by each voter is a negligible piece of information as in order to determine the winner, we do not need to know the ballots cast by each voter, we only need to know their sum ${ }^{7}$. For a considerably wide range of informational environments (balloting procedures) if one wants an aggregation procedure that complies with such a simplicity and with the remaining May's conditions, then using a scoring rule is the only available option.

Second, our result allows to trace the line that separates, in terms of informational simplicity, aggregation procedures that are solely based on the sum of scores from aggregation procedures that are based on the pairwise comparison of the alternatives. Such a line is indeed represented by the fact that, again, scoring rules are the unique procedures that consider anonymously the ballots cast by each voter (in a framework where each voter can cast more than one ballot) and not only the voters.

We also use our informational simplicity argument in favor of scoring rules in

\footnotetext{
${ }^{6}$ For example, under approval balloting a voter might decide to approve as many candidates as she wishes by putting one ballot for each of them in the ballot box. Under Borda balloting the score that a voter assigns to each alternative is equal to the number of ballots she introduces for that specific alternative.

${ }^{7}$ See Palfrey (1989) [28] and Myerson and Weber (1993) [27] for applications in political science.
} 
situations where there is some normatively admissible asymmetry among the alternatives. In a framework where only two alternatives are available Houy (2007) [19] provides an interesting characterization of the qualified majority rule. According to this rule, in order to choose one of the two alternatives, a certain ratio of voters has to support it. Moreover this rule still satisfies Universal Domain, Anonymity and Positive Responsiveness (but obviously fails to satisfy Neutrality). Houy actually proves that the majority rule is characterized by the previous axioms together with two further requirements. The first one is General Abstention according to which if no voter shows up the day of the election then all alternatives win. The second one is Coalition Permanency, the intuition of which is as follows: if a group of abstainers can affect, by voting in a certain way, the outcome of the election, they can do so independently of what the other voters do. We prove that, in our framework, choosing the option whose weighted sum of scores is the highest one (according to a given vector of weights) is the only aggregation procedure that satisfies the five conditions just mentioned.

After providing a short survey of the literature, we describe our setting. We then provide a formal definition of May's axioms in our framework and then we provide a novel characterization of scoring rules. The discussion on the consequences of a weakening of the Neutrality condition and the characterization of weighted scoring rules are relegated to the last section of the paper, prior to the concluding remarks. Most of the technical proofs can be found in the Appendix together with a short analysis of how aggregation procedures based on the pairwise comparison of alternatives can be defined in our informational environment.

\section{Review of the Literature}

May's theorem stands among the few positive results within the literature on voting procedures in which impossibility results abound ${ }^{8}$. In particular, in elections with many alternatives, the difficulty of finding positive results is overwhelming as far as incentives for truth-telling are concerned.

Nonetheless, there are still many studies that, leaving incentives aside, focus on the normative properties of voting procedures, when society has to decide among more than two alternatives. One reason to justify the interest in such properties is that the impossibility results "tell us much about what cannot be done, but leave[s] open the problem of defining what can be done", as Young (1975) [35] puts it.

Scoring rules are a class of voting procedure that has attracted particular interest. The most significant advantage of using scoring rules is probably that they are, in

\footnotetext{
${ }^{8}$ For recent treatments, see Austen-Smith and Banks (1999) [5] and Mccarty and Meirowitz (2006) [24].
} 
general, quite simple to understand. This also explains why they are widely used in real world procedures ${ }^{9}$.

Simple majority rule can be considered as the simplest scoring rule. Several works provide characterization results that are alternative to May's theorem ${ }^{10}$. Other works focus on scoring rules in general. Classical axiomatizations of scoring rules (Smith (1973) [33] and Young (1975) [35]) uniquely consider scoring rules in which a voter can express only a rank ordering of the alternatives. Myerson (1995) [26] drops the ordering assumption and then introduces the notion of generalized scoring rules. Approval voting stands out as the most-well known generalized scoring rule which does not satisfy the ordering condition. More recently, Gilboa and Schmeidler $(2001,2003)[15,16]$ in the context of choice theory provides a more general characterization of scoring rules in which the Neutrality axiom is weakened to the one of Diversity (a condition that ensures the existence of a diverse enough number of scores for the voter $)^{11}$.

All the works mentioned so far do not make any distinction between the balloting procedure and the aggregation procedure. They rather focus on the whole voting procedure with the consequence that the analysis of the informational basis of voting is typically neglected. Importantly, the analysis of Goodin and List (2006) [17] provides a remarkable example of the fact that the normative analysis of an aggregation procedure is affected by the informational structure on which the voting procedure is founded ${ }^{12}$. Our contribution continues along this direction.

\section{The setting}

The set $N=\{1, \ldots, n\}$ is the set of voters while $A=\left\{a_{1}, \ldots, a_{k}\right\}$ is the set of alternatives.

A ballot is a device ${ }^{13}$ used to record choices made by voters. In our model, voters

\footnotetext{
${ }^{9}$ Canada, United Kingdom and United States are, among many others, the most significative examples of countries that still use plurality voting in legislatives elections. approval voting is used by the United Nations General Assembly and several prominent scientific institutions. The Borda Rule is widely used to determine the winner in sport competitions. It is as well used by several scientific institutions and to elect two of the ninety members of the Slovenian National Assembly

${ }^{10}$ See Asan and Sanver (2002) [3], Campbell (1988) [6], Campbell and Kelly (2000) [7]), Cantillon and Rangel (2000) [8], Maskin (1995) [21] and Woeginger (2003) [34]. On a related work, Asan and Sanver (2006) [4] have characterized absolute qualified majority rules in a two-alternatives setting in which Positive Responsiveness is replaced by Maskin Monotonicity.

${ }^{11}$ See Pivato (2011) [29] for a recent work dealing with the roles of Reinforcement and Neutrality in Myerson's framework and Chebotarev and Shamis (1998) [9] for a survey of forty characterizations of scoring rules.

${ }^{12}$ In a recent work, Apesteguia, Ballester and Ferrer (2011) [1] provide a different approach the does not fall in any of the previous categories. The provide an endorsement for scoring rules, by proving that these are the voting procedures that perform best in terms of certain cardinal ideals of justice such as utilitarianism, maximin and maximax.

${ }^{13}$ The term derives from the method used in the seventeenth century to decide the admission
} 
express their opinion through a ballot. Each ballot has on it the name of at most one of the available alternatives. More formally, a ballot is a vector of dimension $|K|$ such that (only) one of its elements ${ }^{14}$ is equal to 1 and all the others are equal to 0. Voters can choose to cast one or more ballots depending on the balloting procedure. A balloting procedure is the set of possible combinations of ballots that a voter is allowed to choose. Each voter can choose one of such combinations. So, for example, if $|A|=3$, under plurality balloting we have

$$
B^{p}=\left\{\left(\begin{array}{ccc}
1 & 0 & 0
\end{array}\right) ;\left(\begin{array}{lll}
0 & 1 & 0
\end{array}\right) ;\left(\begin{array}{lll}
0 & 0 & 1
\end{array}\right)\right\}
$$

whereas under approval balloting, the allowed combinations are as follows:

$$
B^{a}=\left\{\begin{array}{l}
\left(\begin{array}{lll}
1 & 0 & 0 \\
0 & 0 & 0 \\
0 & 0 & 0
\end{array}\right) ;\left(\begin{array}{lll}
0 & 1 & 0 \\
0 & 0 & 0 \\
0 & 0 & 0
\end{array}\right) ;\left(\begin{array}{lll}
0 & 0 & 1 \\
0 & 0 & 0 \\
0 & 0 & 0
\end{array}\right) ; \\
\left(\begin{array}{lll}
1 & 0 & 0 \\
0 & 1 & 0 \\
0 & 0 & 0
\end{array}\right) ;\left(\begin{array}{lll}
0 & 1 & 0 \\
0 & 0 & 1 \\
0 & 0 & 0
\end{array}\right) ; \\
\left(\begin{array}{lll}
1 & 0 & 0 \\
0 & 1 & 0 \\
0 & 0 & 1
\end{array}\right)
\end{array}\right.
$$

More generally, a balloting procedure is a set of matrices of dimension $|A| \times c$ where $c$ is an integer describing the number of ballots that each voter is allowed to cast given the balloting procedure (for example, under plurality balloting $c=1$ ). We consider that the balloting procedure is given and common knowledge to both the voters and the social planner. Even though this is not the only way to represent this class of balloting procedures, the representation we have chosen simplifies the presentation of our axioms.

The vote of each voter $i \in N$ is denoted by $v_{i}$, it simply consists of choosing one (and only one) element from the set specified by the balloting procedure. So, in the second example listed here, a voter who wants to approve of alternatives 1 and 2

of new members in many gentlemen's clubs and similar institutions such as Freemasonry and fraternities. Each voter was supplied with a black and a white ball and he could audibly cast only one of them into the ballot box. The black ball stands as an objection to the admission of the new member whilst the white one stands as a favorable vote. Once the voting procedure was complete the box was opened and the balls displayed. The presence on just one black ball was typically enough to refuse the admission of the new member (Schmidt and Babchuk.[31]).

${ }^{14}$ Our results would remain unaffected if we allowed voters to cast negative scores at the cost of more cumbersome proofs. Furthermore, two scoring rules are identical iff there exists a bijective affine transformation between each of their scores. Hence, any scoring rule including negative scores is equivalent to a scoring rule with positive scores. In what follows, $\mathbb{Q}_{+}$stands for the set of nonnegative rational numbers whereas $\mathbb{N}_{+}$and $\mathbb{R}_{+}$respectively denote the sets of non-negative integers and non-negative real numbers. 
would choose the fourth element in $B^{a}$. This amounts to say that she would insert three ballots in the ballot box, one with the name of alternative 1 on it, the other one with the name of alternative 2 on it and a third one with no name on it. A voter $i \in N$ abstains if she throws in the ballot box $c$ ballots with no name on it.

A vote distribution $v$ represents the list of different choices, given the balloting procedure, made by all the voters taking part to the election. Namely $v=\left(v_{i}\right)_{i \in N}$. By construction $v$ is a matrix composed of $|A|$ columns and $|N| c$ rows. Each of these rows is a ballot cast by some voter so that it has at most one element equal to 1 and all the others equal to 0 . Practically speaking $v$ is the ballot box that contains all the ballots the day of the election. For the ease of the exposition we denote with $\mathcal{N}$ the set of ballots inside the ballot box. Clearly $|\mathcal{N}|=|N| c$. Each entry of the vote distribution is denoted by $v_{q j}$ where $q \in \mathcal{N}$ denotes one of the rows of the matrix and $j$, associated with the alternative $a_{j} \in A$, denotes one of the $|A|$ columns. Let $v^{0}$ denote the vote distribution where every voter decides to abstain. Finally, let $V$ denote the set of all conceivable vote distributions complying with a given balloting procedure. Indeed, the set of vote distributions depends on the given balloting procedure, nonetheless, for the sake of simplicity, we have decided not to make explicit this dependency on the notation.

An aggregation procedure $W: V \rightrightarrows A$ associates every vote distribution $v \in V$ to a nonempty subset of $A$.

For each $a_{j} \in A$, we write $a_{j} \in W(v)$ to denote the fact that such an alternative is in the winning set at the voting distribution $v \in V$. The winner could be unique or there could be several alternatives that are tied.

For all $v \in V$, let the vector $s(v)=\left(s_{a_{1}}(v), s_{a_{2}}(v), \ldots, s_{a_{k}}(v)\right)=\sum_{i=1}^{n} v_{i}$ denote the score vector. Namely, the vector $s(v)$ lists, for each alternative, the total number of ballots, in the ballot box, that stand for that alternative. Finally, let $S(V)$ denote the set of all possible scoring vectors.

\section{May's Axioms}

In what follows, we propose an adaptation of the four conditions proposed by May (1952) [23] to our setting.

May's first condition deals with the richness of the domain of the whole voting procedure. Here we rather impose a condition about the richness of the domain of the aggregation procedure. More precisely we impose no restriction on the set of vote distributions: an aggregation procedure $W$ has to determine a non-empty set of winners for all possible vote distributions $v \in V$.

Universal Domain. The domain of $W$ is the set of all possible vote distributions.

The second condition simply requires that the identity of the voters who has 
cast a certain set of ballots should be irrelevant for the aggregation procedure. Let the mapping $\pi: N \rightarrow N$ denote any of the possible permutations of the voters (that is, any of the possible relabeling of the voters' identity).

Anonymity. For all $u, v \in V$ such that $v=\left(u_{\pi(i)}\right)_{i \in N}, W(u)=W(v)$.

We also propose a stronger version of the previous axiom. The idea is that not only the identity of the voter should be irrelevant for the aggregation procedure. Also the information about the combination of the ballots cast by each voter should be irrelevant for the aggregation procedure. Namely, the fact that a voter has decided to assign a certain score to a certain alternative and, at the same time, some other score to some other alternative should be an irrelevant piece of information for the aggregation procedure. If we restrict our attention to approval balloting this correspond to what Goodin and List (2006)[17] call Optionwise Anonymity. In our more general framework this idea boils down to the fact that the aggregation procedure should only look at the information deriving from a ballot box in which each ballot brings a minimal amount of information (the name of one alternative solely) and such information is absolutely independent from the information provided by the other ballots even if they have been chosen by the same voter. Let the mapping $\sigma: \mathcal{N} \rightarrow \mathcal{N}$ denote any of the possible permutations of the ballots in the ballot box (or, to put it differently any of the possible permutations of the lines of the vote distribution).

Ballot Anonymity. For all $u, v \in V$, such that $v=\sigma(u), W(u)=W(v)$.

The next condition requires to give equal weight to the scores received by any of the alternatives so that if the votes change in such a way that the scores are permuted across alternatives, the outcome is permuted accordingly, i.e "the names of the alternatives do not matter". Let the mapping $\tau: A \rightarrow A$ denote any of the possible permutations of the alternatives (that is, any of the possible relabeling of the alternatives' identity).

Neutrality. For all $u, v \in V$ such that $v=\tau(u)$, for all $a_{j} \in A, a_{j} \in W(u)$ if and only if $a_{\tau(j)} \in W(v)$.

Neutrality imposes quite some structure on the aggregation procedure and, as we will be shown in the section dealing with qualified scoring rules, by relaxing it we are able to characterize a considerably wider family of aggregation procedures. 
Before introducing the next condition, we need to define some notation. For all $u, v \in V$ and $a_{j}, a_{k} \in A, u \succ_{\left\{a_{j} a_{k}\right\}} v$ if and only if there exists a nonempty set of ballots $M \subset \mathcal{N}$ such that

for all $m \in M$, either $u_{m j}>v_{m j}, u_{m k} \leq v_{m k}$ and $u_{m l}=v_{m l}$ for all $l \neq j, k$

$$
\text { or } \quad u_{m j} \geq v_{m j}, u_{m k}<v_{m k} \text { and } u_{m l}=v_{m l} \text { for all } l \neq j, k
$$

for all $m^{\prime} \notin M, u_{m^{\prime}}=v_{m^{\prime}}$.

If a vote distribution changes from $v$ to $u$ then we say that at the vote distribution $u$ the alternative $a_{j}$ dominates the alternative $a_{k}$ with respect to the vote distribution $v$. Informally, $u \succ_{\left\{a_{j} a_{k}\right\}} v$ means that for at least one ballot either the score of option $a_{j}$ increases while the score of some other option $a_{k}$ weakly decreases or the score for option $a_{j}$ weakly increases while the score of $a_{k}$ decreases.

The next axiom requires that the aggregation procedure should be sensitive to such changes of the vote distribution in a specific way. Assume that at some profile $v \in V$ some alternative $a_{j} \in A$ is tied or it is the only winner. Consider a change in the way some voters vote so that we obtain a profile $u \in V$ such that $u \succ_{\left\{a_{j} a_{k}\right\}} v$, for some $a_{k} \in A$. Then, under the new profile option $a_{j}$ must still be a winner whereas $a_{k}$ must not.

Positive Responsiveness. For all $u, v \in V$ and $a_{j}, a_{k} \in A$ such that $a_{j} \in W(v)$ and $u \succ_{\left\{a_{j} a_{k}\right\}} v, a_{j} \in W(u)$ and $a_{k} \notin W(u)$.

Notice that our definition of Positive Responsiveness is slightly stronger than the one proposed by Goodin and List (2006) [17] but it is closer to the one proposed by May (1952) [23]. In the former paper, at the vote distribution $u$ the alternative $a_{j}$ dominates the alternative $a_{k}$ with respect to the vote distribution $v$, i.e. $u \succ_{\left\{a_{j} a_{k}\right\}} v$, if and only if some voters raise the score they assign to alternative $a_{j}$ and, at the same time, they lower the score they give to some alternative $a_{k}$, in other words some voters switch their vote from one alternative to another one. May's concept of domination (like ours) allows, additionally, to consider other tie-breaking situations. Indeed, some voters can also break a tie either because they go from abstention to giving a certain score to some alternative(s) or because they go from giving a certain score to some alternative(s) to abstention. 


\section{Scoring Rules}

If only two alternatives are available May's conditions characterize majority voting. If there are more than two alternatives and voters are allowed to cast just one ballot (plurality balloting), May's conditions characterize plurality voting (as an aggregation procedure). If voters are allowed to cast more than one ballot (i.e., they are allowed to attribute several scores to different alternatives) the class of aggregation procedures that satisfy May's conditions becomes considerably bigger. Let us consider scoring rules first. An aggregation procedure $W$ is a scoring rule if and only if for all $v \in V$ and each $a_{j} \in A$,

$$
a_{j} \in W(v) \text { iff } s_{a_{j}}(v) \geq s_{a_{k}}(v) \text { for all } a_{k} \neq a_{j} .
$$

It is quite easy to see that such an aggregation procedure satisfies all of May's axioms (as defined in the previous section). It satisfies Universal Domain by definition. It satisfies Anonymity because the set of winners depends only on the sum of the votes and not on the identity of the voters. In a similar fashion, it satisfies Neutrality because the set of winners depends on the sum of the votes each alternative gets and not on the name of the alternatives. Finally, it satisfies Positive Responsiveness because if the score of a winning alternative $a_{j}$ is increased by some voters and the score of some other alternative $a_{k}$ is decreased or remains unchanged then $a_{j}$ remains a winning alternative whereas $a_{k}$ necessarily ends up with a score that is strictly lower than $a_{j}$ so that for sure she is not part of the winning set. The argument is the same if the score of $a_{j}$ remains unchanged and the score of $a_{k}$ decreases.

Is this the only aggregation procedure satisfying these axioms? No, it is not. Actually many procedures belonging to the family of procedures based on the pairwise comparison of alternatives, if suitably defined in order to fit our framework, satisfy the axioms we are considering. In our setting the pairwise comparison is based just on the information contained in the ballots and not on the whole voters' preferences rankings. A formal definition, in our framework, of several aggregation procedures based on the pairwise comparison of alternatives is provided in the Appendix.

It turns out that the strengthening Anonymity allows us to create a breach between scoring rules and aggregation procedures based on the pairwise comparisons of alternatives.

Theorem 1. An aggregation procedure satisfies Universal Domain, Ballot Anonymity, Neutrality, and Positive Responsiveness if and only if is a scoring rule.

It is easy to see why a scoring rule satisfies Ballot Anonymity. The outcome of the election solely depends on the total score that each alternative receives which 
ultimately depends on the total amount of ballots cast in favor of a certain option, independently of those cast in favor of others. But this fact also explain why any of the procedures based on the pairwise comparison of alternatives does not satisfy Ballot Anonymity. Consider any two alternatives $a_{j}, a_{k} \in A$. In order to compare these two alternatives we need to be able to know, for each voter, the score she has given to $a_{j}$ and the score she has given to $a_{k}$. That is, for each voter, the information about combination of ballots she has cast is relevant for the aggregation procedure. It is useful to stress that, if voters are allowed to cast at most one ballot (i.e., plurality balloting), the distinction between these two families vanishes. In fact, in this particular informational environment Anonymity and Ballot Anonymity coincide. If more information is asked to the voters (as for example with approval balloting and Borda balloting) then the role of Ballot Anonymity in shaping the additive structure of the balloting procedure becomes essential as shown in the following lemma.

Lemma 1. Let an aggregation procedure $W$ satisfy Ballot Anonymity. Then there exists a $T: S(V) \rightrightarrows A$ such that for all $v \in V, W(v)=T(s(v))$.

Proof. We need to prove that for all $u, v \in V$ such that $s(u)=s(v), W(u)=W(v)$. To see this, it suffices to notice that $s(u)=s(v)$ iff $\sum_{q=1}^{|\mathcal{N}|} u_{q}=\sum_{q=1}^{|\mathcal{N}|} v_{q}$. Moreover, for each row of the vote distribution, $q \in \mathcal{N}$, if we consider the vector $u_{q}$, at most one of its coordinates equals one whereas the rest of them equal zero (the same holds for $v_{q}$ ). Since by assumption $\sum_{q=1}^{|\mathcal{N}|} u_{q}=\sum_{q=1}^{|\mathcal{N}|} v_{q}$, we can define $v$ as a permutation of $u$, that is, $v=\sigma(u)$. So, by Ballot Anonymity, $W(u)=W(v)$. This implies that the aggregation procedure $W$ can be represented by some $T$ such that for all $v \in V$, $W(v)=T(s(v))$.

Lemma 1 says that Ballot Anonymity forces us to summarize the information contained in the ballot box in a very precise way: only the total scores each alternative has been assigned by the voters is relevant, all the extra information eventually contained in the ballot box can be disregarded.

This also means, among other things, that we can restate some of the concepts expressed so far in terms of sum of scores: for each $a_{j}, a_{k} \in A, u \succ_{\left\{a_{j} a_{k}\right\}} v$ if and only if

$$
s_{a_{j}}(u) \geq s_{a_{j}}(v), s_{a_{k}}(u) \leq s_{a_{k}}(v) \text { and } s_{a_{l}}(u)=s_{a_{l}}(v) \text { for all } a_{l} \neq a_{j}, a_{k} ;
$$

with at least one of the inequalities being strict. Moreover if an aggregation procedure $W: V \rightrightarrows A$ also satisfies Neutrality then, for any permutation $\tau$ of the alternatives, for all $a_{j} \in A$

$$
a_{j} \in T(s(v)) \text { if and only if } a_{\tau(j)} \in T(\tau(s(v))),
$$


with $W(v)=T(s(v))$. These observations will be useful for the proof of the following lemma which concludes the proof of Theorem 1.

Lemma 2. Let an aggregation procedure $W: V \rightrightarrows A$ satisfy Universal Domain, Ballot Anonymity, Neutrality and Positive Responsiveness. Then for all $v \in V$ and for all $a_{j} \in A, a_{j} \in W(v)$ iff $s_{a_{j}}(v) \geq s_{a_{k}}(v)$ for all $a_{k} \neq a_{j}$.

Proof. For some $v \in V$ and some $a_{j} \in A$, let $a_{j} \in W(v)$. By lemma $1, W(v)=$ $T(s(v))$ so that $a_{j} \in T(s(v))$. Assume, by way of contradiction, that there exists some $a_{k} \in A$ such that $s_{a_{j}}(v)<s_{a_{k}}(v)$. Let $\tau$ be the permutation that swaps the votes obtained by alternatives $a_{j}$ and $a_{k}$ and leaves the other votes unchanged. This permutation changes accordingly the vector of the total scores. By equation 1 , $a_{\tau(j)}=a_{k} \in T(\tau(s(v)))$. Since by assumption $s_{a_{j}}(v)<s_{a_{k}}(v)$ then, by construction we also have $\tau(v) \succ_{\left\{a_{j} a_{k}\right\}} v$ so that $a_{j} \in T(\tau(s(v)))$ and $a_{k} \notin T(\tau(s(v)))$, the desired contradiction. In order to prove the "only if" part, take any vote distribution $v \in V$ such that $s_{a_{j}}(v) \geq s_{a_{l}}(v)$ for all $a_{l} \neq a_{j}$. Assume, by way of contradiction, that

$a_{j} \notin T\left(s(v)\right.$. As $T(s(v))=W(v) \neq \emptyset$, there must exist some $a_{k} \neq a_{j}$ such that $a_{k} \in T(s(v))$. Let $\tau$ denote again the permutation that swaps the votes obtained by alternative $a_{j}$ and those obtained by alternative $a_{k}$ and leave unchanged the other votes. By equation $1, a_{\tau(j)}=a_{k} \notin T(\tau(s(v)))$. If $s_{a_{j}}(v)=s_{a_{k}}(v)$, then $s(v)=$ $\tau(s(v))$ and thus $a_{k}=a_{\tau(j)} \notin T\left((\tau(s(v)))=T\left((s(v))\right.\right.$, a contradiction. If $s_{a_{j}}(v)>$ $s_{a_{k}}(v)$ then, by construction, $\tau(v) \succ_{\left\{a_{k} a_{j}\right\}} v$ so that, by Positive Responsiveness, $a_{k} \in T(\tau(s(v)))$ which yields, the desired contradiction.

\section{Qualified Scoring Rules}

In this section we study the consequences of a weakening of Neutrality. We are confronted with a wider class of scoring rules in the sense that the aggregation procedure still works by adding the scores deriving from the balloting procedure but these sums can have different weights in order to favor certain alternatives over others. The scoring rules we have proposed in the previous section are just a subfamily of such a family of rules where all the alternatives are treated symmetrically by the aggregation procedure. Indeed the most studied rules such as plurality, Borda and approval voting share the property of being neutral and focusing on voting rules which are not neutral may seem unattractive at first thought. One can think for example of legislative or presidential elections where naturally the alternatives are entitled to receive the same treatment by the voting procedure. However there might be cases where there is some normatively admissible asymmetry among the alternatives as such alternatives are intrinsically different. In such cases society might agree on the fact that they have to be treated differently by the voting procedure. 
This happens often in group decision making when certain alternatives' characteristics such as age, qualification, gender, race may be given a certain weight. In other cases society may also want to bias the collective decision process in favor of the status quo because major changes in some fundamental aspects of the political life should have a wide support. In several countries a qualified majority (or supermajority) is asked in order modify the constitution; the French constitution can be changed if the $60 \%$ of the non-abstaining deputies and senators approve it whereas in India, a two-third majority is needed. Sometimes qualified majorities are part of the legislative procedures in many parliaments. For example in the United States Senate, a three-fifths majority is required to bring out a vote of cloture and avoid obstruction. These examples show that the lack of Neutrality is not unattractive per se. Clearly the choice of the weights to be attached to each alternative always involves some degree of arbitrariness unless this is supported by a specific normative analysis. This is beyond the scope of our paper. ${ }^{15}$

Houy (2007) [19] provides a characterization of the qualified majority rule when voters have to choose between two alternatives ${ }^{16}$. This result can be thought as a generalization of May's theorem as simple majority voting is a particular case of such a rule. Indeed, as for simple majority voting, these rule satisfies Universal Domain, Anonymity and Positive Responsiveness whereas it clearly violate Neutrality. structure.

As in the previous section here we provide a conditional defense of qualified scoring rules when many alternatives are available. That is, assume a society has decided to use a balloting procedure that asks voters to assign some kind of score to the available alternatives. Assume also that such a society does not give the same weight to the alternatives. Then choosing the alternative with the highest weighted sum of scores is the most compelling option. It is the most compelling option in the sense that this aggregation procedure is the only one that satisfies, in our framework, Houy's conditions. The first three conditions used by Houy (2007) [19] are, again, Universal Domain, Anonymity and Positive Responsiveness. The fourth condition, asks to treat neutrally the alternatives only in the extreme case where all the voters decide to abstain. This is a very mild requirement and it is logically implied by Neutrality.

General Abstention. If $v=v^{0}$ then $W(v)=A$.

As stressed already, Neutrality is a fairly strong axiom and plays an important

\footnotetext{
${ }^{15}$ Goodin and List (2006) [18] propose a possible way out from this further ethical puzzle: the symmetrical special-majority. If no option receives the requisite special majority, then no option is chosen.

${ }^{16}$ See also Massó and Vorsatz (2008) [22] for a characterization of weighted approval voting.
} 
role in Theorem 1. By relaxing it we lose the additive structure we are seeking as it might be clear from this simple example that we have adapted from Houy (2007) [19].

Consider only two alternatives and majority balloting. For each $v \in V$, compute the total score vector $s(v)=\left(s_{a_{1}}(v), s_{a_{2}}(v)\right)$. If one of the two alternatives receives a strictly higher score than the other then it is the winner. Otherwise, if

a) $v=v^{0}$ so that $s_{a_{1}}(v)=s_{a_{2}}(v)=0$ then $W(v)=\left(a_{1}, a_{2}\right)$

b) $s_{a_{1}}(v)=s_{a_{2}}(v) \neq 0$. If $s_{a_{1}}(v)$ is odd then $a_{1} \in W(v)$ and $a_{2} \notin W(v)$.

c) $s_{a_{1}}(v)=s_{a_{2}}(v) \neq 0$. If $s_{a_{1}}(v)$ is even then $a_{1} \notin W(v)$ and $a_{2} \in W(v)$.

The example shows that it is possible to find an aggregation procedure that satisfies Universal Domain, Anonymity, Positive Responsiveness and General Abstention but still does not rely entirely on the sum of the scores (weighted or not) to single out the winner of the election. In order to obtain this, Houy (2007) [19] proposes a further condition. This condition focuses on a group of voters who decide to cast one or more ballots with no name on it. If they decide to change their vote in a certain manner they can affect the outcome of the election in a certain way. They should be able to affect the outcome of the election in the same way if they changed their vote in the same way and all the other voters were abstaining.

For any $v \in V$, let $M \subseteq \mathcal{N}$ be a subset of ballots in the vote distribution. Let $v_{M}$ denote the vote distribution we obtain from $v$ when none of the ballots in $M$ has a name on it and the rest of the ballots are as in $v$. Let finally $v_{v \backslash M}$ denote the vote distribution we obtain when the ballots in $M$ are as in $v$ while none of the ballots in $v \backslash M$ has a name on it. Suppose that switching from $v_{M}$ to $v$ alters the outcome of the election, that is, $W\left(v_{M}\right) \neq W(v)$.

Coalition Permanency. For all $v, v_{M}$ and $v_{v \backslash M} \in V$ such that $W\left(v_{M}\right) \neq W(v)$,

$$
W\left(v_{v \backslash M}\right)= \begin{cases}W(v) \backslash W\left(v_{M}\right) & \text { if } W\left(v_{M}\right) \subset W(v) ; \\ W(v) & \text { otherwise. }\end{cases}
$$

Assume that at $v_{M}$ the ballots in a subset $M$ have no name on it. Suppose the voters who have cast those ballots decide to change and use those ballots in favor of certain alternatives. This yields the vote distribution $v$. They can affect the result of the election in two ways: first, they can enlarge the winning set $\left(W\left(v_{M}\right) \subset W(v)\right)$. Coalition Permanency requires that, in such a case, the alternatives which are added to the winning set because of the votes contained in $M$ should be the only ones to win if all the ballots except those in $M$ had no name on it $\left(W\left(v_{v \backslash M}\right)=W(v) \backslash W\left(v_{M}\right)\right)$. Second, they can change the winning set without enlarging it. Coalition Permanency requires that the winners at $v$ should be the same as at $v_{v \backslash M}$. 
Before providing the final result we can provide a more rigorous definition of the family of qualified scoring rules. The aggregation procedure $W$ is a qualified scoring rule if and only if for all $a_{j}, a_{k} \in A$ there exists a strictly positive real number $r_{j k}$, with $r_{k j}=\frac{1}{r_{j k}}$, such that for all $v \in V$

$$
a_{j} \in W(v) \text { iff } s_{a_{j}}(v) \geq r_{j k} s_{a_{k}}(v) \text { for all } a_{k} \neq a_{j} .
$$

It turns out that the five axioms listed so far characterize the whole family of qualified scoring rules.

Theorem 2. An aggregation procedure satisfies Universal Domain, Ballot Anonymity, Positive Responsiveness, General Abstention and Coalition Permanency if and only if it is a qualified scoring rule.

It is simple to understand that any qualified scoring rule satisfies Universal Domain, Anonymity, and Positive Responsiveness. Moreover, by definition it does also satisfy General Abstention. Finally, any qualified scoring rule satisfies Coalition Permanency because if a group of voters is able to change the outcome the election by raising by a certain amount the scores given to certain alternatives (given the vote of all the other members of the population), then these (marginal) scores alone would lead to the victory of such alternatives if all the other members of the population were abstaining. The proof of theorem 2 is quite involved and it is relegated to the Appendix.

\section{Concluding Comments}

This work presents a novel rationale for scoring rules as it relates this family of voting rules to the conditions of the celebrated May's Theorem. We give particular emphasis to the informational simplicity of scoring rules (or, to put it differently, the amount of the information deriving from the balloting procedure they take into account). This is described by a particular condition we impose on the aggregation procedure, Ballot Anonymity, a condition that can be understood as follows. In our framework voters are allowed to cast more than one ballot, each ballot standing for one alternative. Ballot Anonymity requires the aggregation procedure to consider as irrelevant the specific information about the particular combination of ballots cast by each voter. It turns out that this condition is essential in characterizing scoring rules together with the other conditions of May's theorem: Universal Domain, Positive Responsiveness and Neutrality. This result is also prone to an alternative interpretation: Ballot Anonymity represents the divide between scoring rules and aggregation procedures based on the pairwise comparison of alternatives. This pro- 
vides a better understanding of the informational foundations of both families of rules.

As already stressed our work is closely related to Goodin and List (2006) [17]. The main difference between our contribution and theirs is that we formally disentangle the different combinations of ballots a voter might choose (given by the balloting procedure) from the input of the aggregation procedure (the collection of ballots contained in the ballot box). This allows us to enlarge significantly the scope of the axiomatization of the aggregation procedures. Indeed we are able to provide the May-like conditions that characterize any scoring rule.

Our characterization is shown to be robust to situations where some normatively admissible asymmetry between alternatives is considered. Readapting to our informational framework the conditions proposed by Houy (2007) [19] to characterize the qualified majority rule (in the two-option case) we provide a full characterization of qualified scoring rules.

\section{Appendix - Aggregation procedures based on the pair- wise comparisons of alternatives}

In this section we provide a definition of some aggregation procedures based on the pairwise comparisons of alternatives, the Condorcet rules. Usually such a comparison is based on the whole ranking over alternatives that is asked to each voter. We adapt the procedures to our framework in the sense that the pairwise comparison is based on the information that is made available by the balloting procedure. The definitions are adaptations of those one can find in Fishburn (1977) [12] and Laslier (1997) [20].

For each $m \in N$ and $a_{i} \in A$, let $s_{m}\left(a_{i}, v\right)$ denote the score that voter $m$ has assigns to alternative $a_{i}$ at the vote distribution $v \in V$. In our framework this amounts to count the number of ballots that this voter has cast in favor of alternative $a_{i}$. For all $v \in V$, and for any pair of alternatives $a_{i}, a_{j} \in A$, let $p\left(a_{i}, a_{j}, v\right)$ be the number of voters which assign a higher score to alternative $a_{i}$ than to alternative $a_{j}$, i.e.

$$
p\left(a_{i}, a_{j}, v\right)=\#\left\{m \in N \mid v_{m i} \geq v_{m j}\right\} .
$$

The pairwise comparison of the alternatives $a_{i}, a_{j} \in A$ is based on such scores. We say that $a_{i}$ is not defeated by $a_{j}$ at the profile $v \in V$, noted $a_{i} D_{v} a_{j}$, if and only if $p\left(a_{i}, a_{j}, v\right) \geq p\left(a_{j}, a_{i}, v\right)$ (alternatives $a_{i}$ and $a_{j}$ are tied at the pairwise comparison if $\left.p\left(a_{i}, a_{j}, v\right)=p\left(a_{j}, a_{i}, v\right)\right)$.

Moreover, for all $v \in V$ and $a_{i} \in A$, we denote:

- $b\left(a_{i}, v\right)=\sum_{a_{j} \in A} p\left(a_{i}, a_{j}, v\right)$

- $c\left(a_{i}, v\right)=\#\left\{a_{j} \in A: a_{i} D_{v} a_{j}\right\}-\#\left\{a_{j} \in A: a_{j} D_{v} a_{i}\right\}$; 
- $d\left(a_{i}, v\right)=$ number of voters in the largest subset from $v$ for which $a_{i}$ ties or beats every other alternatives on the basis of simple majority with respect to this subset. If there is no such subset then $d\left(a_{i}, v\right)=0$;

- $e\left(a_{i}, v\right)=\min _{a_{j} \in A \backslash\left\{a_{i}\right\}} p\left(a_{i}, a_{j}, v\right)$.

Black's rule. For all $v \in V, W_{B}(v)=\left\{a_{i} \in A: a_{j} D_{v} a_{i}\right.$ for no $\left.a_{j} \in A\right\}$ if the latter set is nonempty. Otherwise $W$ is determined by Borda count over the vote distribution:

$$
W_{B}(v)=\left\{a_{i} \in A: b\left(a_{i}, v\right) \geq b\left(a_{j}, v\right) \text { for all } a_{j} \in A\right\} .
$$

Copeland's Rule. For all $v \in V, W_{C o p}(v)=\left\{a_{i} \in A: c\left(a_{i}, v\right) \geq c\left(a_{j}, v\right)\right.$ for all $a_{j} \in$ $A\}$.

Young's rule. For all $v \in V$ and $d\left(a_{i}, v\right)$, let $d^{*}\left(a_{i}, v\right)=\lim _{n \rightarrow \infty} d\left(a_{i}, n v\right) / n$. Then

$$
W_{\text {You }}(v)=\left\{a_{i} \in A: d^{*}\left(a_{i}, v\right) \geq d^{*}\left(a_{j}, v\right) \text { for all } a_{j} \in A\right\} .
$$

Note that $n V$, for any integer $n$, stands for the fact the vote distribution $v$ being replicated $n$ times.

Condorcet's rule (or Minimax function). For all $v \in V$,

$$
W_{\text {Con }}(v)=\left\{a_{i} \in A: e\left(a_{i}, v\right) \geq e\left(a_{j}, v\right) \text { for all } a_{j} \in A\right\}
$$

Kemeny's rule. For all $v \in V$, let $L^{*}$ be the collection of ballots $L$ that maximize $\sum_{a_{i} \neq a_{j}} s\left(a_{i}, a_{j}\right) L\left(a_{i}, a_{j}\right)$, where $L\left(a_{i}, a_{j}\right)=1$ if $b\left(a_{i}, v\right) \geq b\left(a_{j}, v\right)$, and $L\left(a_{i}, a_{j}\right)=0$ otherwise. Then

$$
W_{K e m}(v)=\left\{a_{i} \in A: \text { there is an } L \in L^{*} \text { such that } a_{i} L a_{j} \text { for all } a_{j} \in A\right\}
$$

Remark 1. Under Approval, Borda and Plurality Balloting, all the rules listed here satisfy Universal Domain, Anonymity, Neutrality and Positive Responsiveness. They all fail to satisfy Ballot Anonimity.

\section{Appendix - Proof of Theorem 2}

Let an aggregation procedure $W$ satisfy all the listed conditions. The following lemmata, together with Lemma 1 will be useful for the final proof. We define for any strictly positive integer $p, p / 0=\infty$

Lemma 3. Let an aggregation procedure $W: V \rightrightarrows A$ satisfy Universal Domain, Positive Responsiveness and General Abstention. Then, for all $a_{j}, a_{k} \in A$ and all $v \in V$ such that $\frac{s_{a_{j}}(v)}{s_{a_{k}}(v)}=0, a_{j} \notin W(v)$. 
Proof. For all $v \in V$ let $A^{0}(v)=\left\{a_{j} \in A \mid s_{a_{j}}(v)=0\right\}$. Clearly at $v^{0} \in V,\left|A^{0}\left(v^{0}\right)\right|=$ $|A|$. By General Abstention, $W\left(v^{0}\right)=A$. Let us consider all $v^{1} \in V$ such that, for one and only one alternative $a_{k} \in A, s_{a_{k}}\left(v^{1}\right)>0$ so that $\left|A^{0}\left(v^{1}\right)\right|=|A|-1$. Let us assume that, for some $a_{j} \neq a_{k}, a_{j} \in W\left(v^{1}\right)$. By construction $v^{1} \succ_{\left\{a_{k} a_{j}\right\}} v^{0}$ so that, by Positive Responsiveness, $a_{j} \notin W\left(v^{1}\right)$, a contradiction.

Let us consider now a vote distribution $v^{d} \in V$ such that $\left|A^{0}\left(v^{d}\right)\right|=|A|-d$ with $1<d<|A|$. Let us assume that, for some $a_{j} \in A^{0}\left(v^{d}\right), a_{j} \in W\left(v^{d}\right)$. Take now some alternative $h \in A \backslash A^{0}\left(v^{d}\right)$ and consider some vote distribution $v^{d-1} \in V$ such that $s_{a_{h}}\left(v^{d-1}\right)=0$ and $s_{a_{l}}\left(v^{d-1}\right)=s_{a_{l}}\left(v^{d}\right)$ for all $a_{h} \neq a_{l}$. By construction $v^{d-1} \succ_{\left\{a_{j} a_{h}\right\}}$ $v^{d}$ since $s_{a_{j}}\left(v^{d-1}\right) \geq s_{a_{j}}\left(v^{d}\right)=0,0=s_{a_{h}}\left(v^{d-1}\right)<s_{a_{h}}\left(v^{d}\right)$ and $s_{a_{l}}\left(v^{d-1}\right)=s_{a_{l}}\left(v^{d}\right)$ for all $a_{l} \neq a_{j}, a_{h}$. Hence, by Positive Responsiveness $a_{j} \in W\left(v^{d-1}\right)$. Iterating this process leads to a vote distribution $v^{1}$ in which for only one alternative $a_{k}\left(a_{k} \neq a_{j}\right)$, $s_{a_{k}}\left(v^{1}\right)>0$ but still $a_{j} \in W\left(v^{1}\right)$, the desired contradiction.

Fix some alternative $a_{k} \in A$. For each $a_{j} \in A$ let $r_{a_{j}}=\frac{s_{a_{j}}(v)}{s_{a_{k}}(v)}$ and $r=\left\{r_{a_{j}}\right\}_{a_{j} \in A} \in$ $\mathbb{Q}^{|A|}$. Finally let $V^{r}$ denote the family of vote distributions whose score ratios are equal to $r$; i.e.,

$$
V^{r}=\left\{v \in V \mid \text { for all } a_{j} \in A, \frac{s_{a_{j}}(v)}{s_{a_{k}}(v)}=r_{a_{j}}\right\} .
$$

Lemma 4. Let $r=\left\{r_{a_{j}}\right\}_{a_{j} \in A} \in \mathbb{Q}^{|A|}$. There exists some $v^{*} \in V^{r}$ such that, for all $v \in V^{r}$,

$$
s(v)=\left(r_{a_{1}}, \ldots, r_{a_{k-1}}, 1\right) m s_{a_{k}}\left(v^{*}\right)
$$

with $m \in \mathbb{N}_{+}$.

Proof. Let us pick some $r=\left\{r_{a_{j}}\right\}_{a_{j} \in A}$ with $0<r_{a_{j}}<\infty$ for each $a_{j} \in A$. Let us consider the set $V^{r}=\left\{v \in V \mid\right.$ for all $\left.a_{j} \in A, \frac{s_{a_{j}}(v)}{s_{a_{k}}(v)}=r_{a_{j}}\right\}$. Take the vote distribution $v^{*} \in V^{r}$ such that, for all $v \in V^{r}$ and for all $a_{j} \in A, s_{a_{j}}\left(v^{*}\right) \leq s_{a_{j}}(v)$. By construction $s\left(v^{*}\right)=r s_{a_{k}}\left(v^{*}\right)$ so that, for all $a_{j} \in A, r_{a_{j}} s_{a_{k}}\left(v^{*}\right)$ is an integer. Moreover for all $m \in \mathbb{N}_{+}$and for all $a_{j} \in A, m r_{a_{j}} s_{a_{k}}\left(v^{*}\right)$ is still an integer so that for all $v \in V$ such that $s(v)=m s\left(v^{*}\right), v \in V^{r}$.

Assume, by way of contradiction that there exists $v^{1} \in V^{r}$ and some strictly positive $\widehat{m} \in \mathbb{R}_{+} \backslash \mathbb{N}_{+}$such that $s\left(v^{1}\right)=\widehat{m} s\left(v^{*}\right)$. Let $|m|$ be the greatest integer such that $|m|<\widehat{m}$. This implies that $0<\widehat{m}-|m|<1$. Take $v^{2}, v^{3} \in V$ such that

$$
s\left(v^{2}\right)=|m| s\left(v^{*}\right)
$$

so that $v^{2} \in V^{r}$ and

$$
s\left(v^{3}\right)=s\left(v^{1}\right)-s\left(v^{2}\right) .
$$


Hence, for each $a_{j} \in A$,

$$
s_{a_{j}}\left(v^{3}\right)=s_{a_{j}}\left(v^{1}\right)-s_{a_{j}}\left(v^{2}\right)=\widehat{m} s_{a_{j}}\left(v^{*}\right)-|m| s_{a_{j}}\left(v^{*}\right)=(\widehat{m}-|m|) s_{a_{j}}\left(v^{*}\right)<s_{a_{j}}\left(v^{*}\right)
$$

and

$$
\frac{s_{a_{j}}\left(v^{3}\right)}{s_{a_{k}}\left(v^{3}\right)}=\frac{(\widehat{m}-|m|)\left(s_{a_{j}}\left(v^{*}\right)\right)}{(\widehat{m}-|m|)\left(s_{a_{k}}\left(v^{*}\right)\right)}=r_{a_{j}} .
$$

From equation (3), $v^{3} \in V^{r}$ but this, in light of equation (2), contradicts the definition of $v^{*}$. For the case in which, for some $a_{j} \in A$, either $r_{a_{j}}=0$ or $r_{a_{j}}=\infty$ the same proof applies, we just have to restrict our attention to the alternatives such that $0<r_{a_{j}}<\infty$.

Lemma 5. Let an aggregation procedure $W: V \rightrightarrows A$ satisfy Universal Domain, Anonymity, Coalition Permanency and General Abstention. Then, for all $v^{1}, v^{2} \in$ $V_{b}^{r}, W\left(v^{1}\right)=W\left(v^{2}\right)$.

Proof. By lemma 1, for all $v \in V, W(v)=T(s(v))$. By lemma 4 there exists some $v^{\prime \prime} \in V_{b}^{r}$ such that for all $v^{1} \in V_{b}^{r}, s\left(v^{1}\right)=m s\left(v^{\prime \prime}\right)$ where $m \in \mathbb{N}_{+}$. Assume that $T\left(s\left(v^{1}\right)\right) \neq T\left(s\left(v^{\prime \prime}\right)\right)$. If $m=1$ we have a contradiction with Lemma 1 . Let $m>1$ so that

$$
s\left(v^{1}\right)=m s\left(v^{\prime \prime}\right)=s\left(v^{\prime \prime}\right)+(m-1) s\left(v^{\prime \prime}\right) .
$$

Within the rest of the proof, we work with the function $T$ rather than with $W$, to ease the presentation of the result. By slight abuse of notation, we use $(m-j) s\left(v^{\prime \prime}\right)$ in order to refer to the vote distribution $v^{j}$ which score vector equals $(m-j) s\left(v^{\prime \prime}\right)$.

Since by assumption $T\left(s\left(v^{1}\right)\right) \neq T\left(s\left(v^{\prime \prime}\right)\right)$ then, by Coalition Permanency

$T\left((m-1) s\left(v^{\prime \prime}\right)\right)= \begin{cases}T\left(m s\left(v^{\prime \prime}\right)\right) \backslash T\left(s\left(v^{\prime \prime}\right)\right) & \text { if } T\left(s\left(v^{\prime \prime}\right)\right) \subset T\left(m s\left(v^{\prime \prime}\right)\right), \\ T\left(m s\left(v^{\prime \prime}\right)\right) & \text { otherwise. }\end{cases}$

Suppose first that $T\left(s\left(v^{\prime \prime}\right)\right) \not \subset T\left(m s\left(v^{\prime \prime}\right)\right)$ so that $T\left((m-1) s\left(v^{\prime \prime}\right)\right)=T\left(m s\left(v^{\prime \prime}\right)\right) \neq$ $T\left(s\left(v^{\prime \prime}\right)\right)$. Again, by Coalition Permanency :

$T\left((m-2) s\left(v^{\prime \prime}\right)\right)= \begin{cases}T\left((m-1) s\left(v^{\prime \prime}\right)\right) \backslash T\left(s\left(v^{\prime \prime}\right)\right) & \text { if } T\left(s\left(v^{\prime \prime}\right)\right) \subset T\left((m-1) s\left(v^{\prime \prime}\right)\right), \\ T\left((m-1) s\left(v^{\prime \prime}\right)\right) & \text { otherwise. }\end{cases}$

From the previous step, $T\left((m-1) s\left(v^{\prime \prime}\right)\right)=T\left(m s\left(v^{\prime \prime}\right)\right)$ and $T\left(s\left(v^{\prime \prime}\right)\right) \not \subset T\left(m s\left(v^{\prime \prime}\right)\right)$ so that $T\left((m-2) s\left(v^{\prime \prime}\right)\right)=T\left((m-1) s\left(v^{\prime \prime}\right)\right)$. Repeating the same argument we arrive to

$$
T\left(s\left(v^{\prime \prime}\right)\right)=T\left(2 s\left(v^{\prime \prime}\right)\right)=, \ldots,=T\left(m s\left(v^{\prime \prime}\right)\right)=T\left(s\left(v^{1}\right)\right),
$$


contradicting the assumption that $T\left(s\left(v^{1}\right)\right) \neq T\left(s\left(v^{\prime \prime}\right)\right)$.

Suppose now that $T\left(s\left(v^{\prime \prime}\right)\right) \subset T\left(m s\left(v^{\prime \prime}\right)\right)$ so that $T\left((m-1) s\left(v^{\prime \prime}\right)\right)=T\left(m s\left(v^{\prime \prime}\right)\right) \backslash T\left(s\left(v^{\prime \prime}\right)\right)$. From this it follows that $T\left((m-1) s\left(v^{\prime \prime}\right)\right) \neq T\left(s\left(v^{\prime \prime}\right)\right)$. Hence, by Coalition Permanency

$T\left((m-2) s\left(v^{\prime \prime}\right)\right)= \begin{cases}T\left((m-1) s\left(v^{\prime \prime}\right)\right) \backslash T\left(s\left(v^{\prime \prime}\right)\right) & \text { if } T\left(s\left(v^{\prime \prime}\right)\right) \subset T\left((m-1) s\left(v^{\prime \prime}\right)\right), \\ T\left((m-1) s\left(v^{\prime \prime}\right)\right) & \text { otherwise. }\end{cases}$

From the first step we know that $T\left((m-1) v^{\prime \prime}\right)=T\left(m s\left(v^{\prime \prime}\right)\right) \backslash T\left(s\left(v^{\prime \prime}\right)\right)$ so that $T\left(s\left(v^{\prime \prime}\right)\right) \not \subset T\left((m-1) s\left(v^{\prime \prime}\right)\right)$. Hence

$$
T\left((m-2) s\left(v^{\prime \prime}\right)\right)=T\left((m-1) s\left(v^{\prime \prime}\right)\right)=T\left(m s\left(v^{\prime \prime}\right)\right) \backslash T\left(s\left(v^{\prime \prime}\right)\right) .
$$

Repeating the same argument we are again lead to

$$
T\left(s\left(v^{\prime \prime}\right)\right)=T\left(2 s\left(v^{\prime \prime}\right)\right)=, \ldots,=T\left(m s\left(v^{\prime \prime}\right)\right) \backslash T\left(s\left(v^{\prime \prime}\right)\right)
$$

which yields the desired contradiction. Hence, we can write that for any $v^{1} \in V_{b}^{r}$

$$
T\left(s\left(v^{\prime \prime}\right)\right)=T\left(s\left(v^{1}\right)\right) .
$$

For all $v^{1}, v^{2} \in V$ such that, for all $a_{i} \in A s_{a_{i}}\left(v^{1}\right) \geq s_{a_{i}}\left(v^{2}\right)$, let $v^{1}-v^{2} \in V^{b}$ denote the vote distribution such that, for all $a_{i} \in A$,

$$
s_{a_{i}}\left(v^{1}-v^{2}\right)=s_{a_{i}}\left(v^{1}\right)-s_{a_{i}}\left(v^{2}\right) \geq 0 .
$$

Lemma 6. Let an aggregation procedure $W: V \rightrightarrows A$ satisfy Anonymity, Positive Responsiveness, General Abstention and Coalition Permanency. Let $v^{1}, v^{2} \in V$ and $a_{i}, a_{j} \in A$ be such that $a_{i}, a_{j} \in W\left(v^{1}\right) \cap W\left(v^{2}\right)$. Let moreover $s_{a_{i}}\left(v^{1}\right)>s_{a_{i}}\left(v^{2}\right)$, $s_{a_{j}}\left(v^{1}\right)>s_{a_{j}}\left(v^{2}\right)$ and $s_{a_{k}}\left(v^{1}\right)=s_{a_{k}}\left(v^{2}\right)$ for all $a_{k} \neq a_{i}, a_{j}$. Then $W\left(v^{1}-v^{2}\right)=$ $\left\{a_{i}, a_{j}\right\}$.

Proof. Let $v^{1}, v^{2} \in V$ denote a pair of vote distributions such that $a_{i}, a_{j} \in W\left(v^{1}\right) \cap$ $W\left(v^{2}\right)$ for some $a_{i}, a_{j} \in A$. Furthermore, let $s_{a_{i}}\left(v^{1}\right)>s_{a_{i}}\left(v^{2}\right), s_{a_{j}}\left(v^{1}\right)>s_{a_{j}}\left(v^{2}\right)$ and $s_{a_{k}}\left(v^{1}\right)=s_{a_{k}}\left(v^{2}\right)$ for all $a_{k} \neq a_{i}, a_{j}$. Hence, the vote distribution $v^{1}-v^{2} \in V^{b}$ is well-defined. Indeed, it satisfies $s_{a_{k}}\left(v^{1}-v^{2}\right)=s_{a_{k}}\left(v^{1}\right)-s_{a_{k}}\left(v^{2}\right)=0$ for all $a_{k} \neq a_{i}, a_{j}$ and that both $s_{a_{i}}\left(v^{1}-v^{2}\right)$ and $s_{a_{j}}\left(v^{1}-v^{2}\right)$ are strictly positive. Furthermore, Lemma 3 implies that every alternative $a_{k} \neq a_{i}, a_{j}$ does not belong to $W\left(v^{1}-v^{2}\right)$; in other words $W\left(v^{1}-v^{2}\right) \subseteq\left\{a_{i}, a_{j}\right\}$.

Let us assume by contradiction that $W\left(v^{1}-v^{2}\right) \subset\left\{a_{i}, a_{j}\right\}$. This implies that 
$W\left(v^{1}\right) \neq W\left(v^{1}-v^{2}\right)$ so that by $C P$, we can write

$W\left(v^{2}\right)= \begin{cases}W\left(v^{1}-v^{2}+v^{2}\right) \backslash W\left(v^{1}-v^{2}\right)=W\left(v^{1}\right) \backslash W\left(v^{1}-v^{2}\right) & \text { if } W\left(v^{1}-v^{2}\right) \subset W\left(v^{1}\right), \\ W\left(v^{1}-v^{2}+v^{2}\right)=W\left(v^{1}\right) & \text { otherwise. }\end{cases}$

However, $W\left(v^{1}-v^{2}\right) \subset\left\{a_{i}, a_{j}\right\} \subseteq W\left(v^{1}\right)$, then $W\left(v^{2}\right)=W\left(v^{1}\right) \backslash W\left(v^{1}-v^{2}\right)$.

However, as by assumption $W\left(v^{1}-v^{2}\right) \subset\left\{a_{i}, a_{j}\right\}$, previous equality implies that

either $a_{i}$ or $a_{j}$ does not belong to $W\left(v^{2}\right)$, a contradiction.

Lemma 7. Let an aggregation procedure $W: V \rightrightarrows A$ satisfy Universal Domain, Anonymity, Positive Responsiveness, General Abstention and Coalition Permanency. For all $v \in V \backslash v^{0}$ and $a_{i}, a_{j} \in A$ such that $a_{i}, a_{j} \in W(v)$, there is at most one $r \in \mathbb{Q}_{+}$ such that $\frac{s_{a_{i}}(v)}{s_{a_{j}}(v)}=r$.

Proof. Let

$$
R^{i j}=\left\{r \in \mathbb{Q}_{+} \mid \exists v \in V \text { s.t. } \frac{s_{a_{i}}(v)}{s_{a_{j}}(v)}=r \text { and } a_{i}, a_{j} \in W(v)\right\}
$$

Assume by contradiction that $\left|R^{i j}\right| \geq 2$. Let $r^{+}=\max R^{i j}$ and $r^{-}=\min R^{i j}$, since $N$ is finite then $r^{+}$and $r^{-}$are well defined. Moreover, by Lemma $3 r^{-}>0$ and $r^{+}<\infty$. Let also

$$
E^{+}=\left\{v \in V \mid \frac{s_{a_{i}}(v)}{s_{a_{j}}(v)}=r^{+} \text {and } a_{i}, a_{j} \in W(v)\right\}
$$

and

$$
E^{-}=\left\{v \in V \mid \frac{s_{a_{i}}(v)}{s_{a_{j}}(v)}=r^{-} \text {and } a_{i}, a_{j} \in W(v)\right\}
$$

Finally let us take some vote distribution $v^{1} \in E^{+}$and $v^{2} \in E^{-}$such that $s_{a_{k}}\left(v^{1}\right)=s_{a_{k}}\left(v^{2}\right)$ for all $a_{k} \neq a_{i}, a_{j}$. If both $E^{+}$and $E^{-}$are non-empty (otherwise the claim is proved), it is always possible to find such two vectors. Indeed, consider any $v$ in $E^{+}$. Take the vote distribution $v^{1}$ such that $s_{a_{i}}\left(v^{1}\right)=s_{a_{i}}(v)$, $s_{a_{j}}\left(v^{1}\right)=s_{a_{j}}(v)$ and $s_{a_{k}}\left(v^{1}\right)=0$ for all $a_{k} \neq a_{i}, a_{j}$. By construction, we can write $v^{1} \succ_{\left\{a_{i} a_{h}\right\}} v$ for each $a_{h} \neq a_{i}, a_{j}$ and $v^{1} \succ_{\left\{a_{j} a_{h}\right\}} v$ for each $a_{h} \neq a_{i}, a_{j}$ so that Positive Responsiveness implies that $v^{1} \in E^{+17}$. A similar argument applies to any vote distribution $v$ in $E^{-}$. Again it suffices to take a vote distribution $v^{2} \in E^{-}$ with $s_{a_{i}}\left(v^{2}\right)=s_{a_{i}}(v), s_{a_{j}}\left(v^{2}\right)=s_{a_{j}}(v)$ and $s_{a_{k}}\left(v^{2}\right)=0$ for all $a_{k} \neq a_{i}, a_{j}$. Clearly

\footnotetext{
${ }^{17}$ Positive Responsiveness does not directly imply it, even though it does it indirectly. One has to define a sequence of intermediate vote distributions, in the spirit of the proof of Lemma 3. For instance, take $v^{\prime}$ with $s_{a_{i}}\left(v^{\prime}\right)=s_{a_{i}}(v)$ for $a_{i} \neq a_{h}$ and $s_{a_{h}}\left(v^{\prime}\right)=0$. It is then immediate that, by Positive Responsiveness, $v^{\prime} \in E^{+}$. One has to iterate the same procedure on the different alternatives to build the vote distribution $v^{1}$.
} 
$s_{a_{k}}\left(v^{1}\right)=s_{a_{k}}\left(v^{2}\right)$ for all $a_{k} \neq a_{i}, a_{j}$ so that $v^{1}$ and $v^{2}$ exist.

We have that by assumption $\frac{s_{a_{i}}\left(v^{1}\right)}{s_{a_{j}}\left(v^{1}\right)}=r^{+}>r^{-}=\frac{s_{a_{i}}\left(v^{2}\right)}{s_{a_{j}}\left(v^{2}\right)}$ so that the following cases arise:

$1-s_{a_{i}}\left(v^{1}\right)>s_{a_{i}}\left(v^{2}\right)$ and $s_{a_{j}}\left(v^{1}\right) \leq s_{a_{j}}\left(v^{2}\right)$ (or $s_{a_{i}}\left(v^{1}\right) \geq s_{a_{i}}\left(v^{2}\right)$ and $s_{a_{j}}\left(v^{1}\right)<$ $\left.s_{a_{j}}\left(v^{2}\right)\right)$. This yields a contradiction with Positive Responsiveness as by definition $v^{1} \succ_{\left\{a_{i} a_{j}\right\}} v^{2}$ implying that $a_{j} \notin W\left(v^{2}\right)$.

$2-s_{a_{i}}\left(v^{1}\right) \leq s_{a_{i}}\left(v^{2}\right)$ and $s_{a_{j}}\left(v^{1}\right) \geq s_{a_{j}}\left(v^{2}\right)$. This yields a contradiction with the assumption that $r^{-}<r^{+}$.

$3-s_{a_{i}}\left(v^{1}\right)<s_{a_{i}}\left(v^{2}\right)$ and $s_{a_{j}}\left(v^{1}\right)<s_{a_{j}}\left(v^{2}\right)$. In this case, the vote distribution $v^{2}-v^{1}$ can be defined. By Lemma 6, we know that $W\left(v^{2}-v^{1}\right)=\left\{a_{i}, a_{j}\right\}$. However, one can prove that $s_{a_{i}}\left(v^{2}-v^{1}\right) / s_{a_{j}}\left(v^{2}-v^{1}\right)<r^{-}$which entails a contradiction with the definition of $r^{-}$.

$4-s_{a_{i}}\left(v^{1}\right)>s_{a_{i}}\left(v^{2}\right)$ and $s_{a_{j}}\left(v^{1}\right)>s_{a_{j}}\left(v^{2}\right)$. In this case, the vote distribution $v^{1}-v^{2}$ can be defined. By Lemma 6 , we know that $W\left(v^{1}-v^{2}\right)=\left\{a_{i}, a_{j}\right\}$. However, one can prove that $s_{a_{i}}\left(v^{1}-v^{2}\right) / s_{a_{j}}\left(v^{1}-v^{2}\right)>r^{+}$which entails a contradiction with the definition of $r^{+}$.

Lemma 8. Let an aggregation procedure $W: V \rightrightarrows A$ satisfy Universal Domain, Anonymity, Positive Responsiveness, General Abstention and Coalition Permanency. For all $v^{1}, v^{2} \in V \backslash v^{0}$ such that $\frac{s_{a_{i}}\left(v^{1}\right)}{s_{a_{j}}\left(v^{1}\right)}>\frac{s_{a_{i}}\left(v^{2}\right)}{s_{a_{j}}\left(v^{2}\right)}$ for some couple of alternatives $a_{i}, a_{j} \in A$

$$
a_{i} \in W\left(v^{2}\right) \Longrightarrow a_{j} \notin W\left(v^{1}\right) \text { and } a_{j} \in W\left(v^{1}\right) \Longrightarrow a_{i} \notin W\left(v^{2}\right) .
$$

Proof. Let $v^{1}, v^{2} \in V \backslash v^{0}$ and $a_{i}, a_{j} \in A$ be such that $\frac{s_{a_{i}}\left(v^{1}\right)}{s_{a_{j}}\left(v^{1}\right)}>\frac{s_{a_{i}}\left(v^{2}\right)}{s_{a_{j}}\left(v^{2}\right)}$. We only prove the first part of the statement, $a_{i} \in W\left(v^{2}\right) \Longrightarrow a_{j} \notin W\left(v^{1}\right)$. A similar argument proves that $a_{j} \in W\left(v^{1}\right) \Longrightarrow a_{i} \notin W\left(v^{2}\right)$. Assume for the sake of contradiction that $a_{i} \in W\left(v^{2}\right) \Longrightarrow a_{j} \in W\left(v^{1}\right)$.

Let

$$
R^{j}=\left\{r \in \mathbb{Q}_{+} \mid \exists v \in V \text { s.t. } \frac{s_{a_{i}}(v)}{s_{a_{j}}(v)}=r \text { and } a_{j} \in W(v)\right\}
$$

and

$$
R^{i}=\left\{r \in \mathbb{Q}_{+} \mid \exists v \in V \text { s.t. } \frac{s_{a_{i}}(v)}{s_{a_{j}}(v)}=r \text { and } a_{i} \in W(v)\right\} .
$$

Let $r^{+}=\max R^{j}$ and $r^{-}=\min R^{i}$. Since $N$ is finite, both $r^{+}$and $r^{-}$are well defined and, by Lemma $3, r^{+}<\infty$ and $r^{-}>0$. Moreover, by assumption, $\frac{s_{a_{i}}\left(v^{1}\right)}{s_{a_{j}}\left(v^{1}\right)} \leq r^{+}$and $\frac{s_{a_{i}}\left(v^{2}\right)}{s_{a_{j}}\left(v^{2}\right)} \geq r^{-}$so that $r^{+}>r^{-}$. Let also

$$
F^{+}=\left\{v \in V \mid \frac{s_{a_{i}}(v)}{s_{a_{j}}(v)}=r^{+} \text {and } a_{j} \in W(v)\right\}
$$


and

$$
F^{-}=\left\{v \in V \mid \frac{s_{a_{i}}(v)}{s_{a_{j}}(v)}=r^{-} \text {and } a_{i} \in W(v)\right\} .
$$

Let us take $\widehat{v}^{1} \in F^{+}$and $\widehat{v}^{2} \in F^{-}$such that $s_{a_{k}}\left(\widehat{v}^{1}\right)=s_{a_{k}}\left(\widehat{v}^{2}\right)$ for all $a_{k} \neq a_{i}, a_{j}$. An argument similar to the one used in the proof of lemma 7 proves that two such vectors always exist.

We can now consider the following cases:

$1-s_{a_{i}}\left(\widehat{v}^{1}\right)>s_{a_{i}}\left(\widehat{v}^{2}\right)$ and $s_{a_{j}}\left(\widehat{v}^{1}\right) \leq s_{a_{j}}\left(\widehat{v}^{2}\right)\left(\right.$ or $s_{a_{i}}\left(\widehat{v}^{1}\right) \geq s_{a_{i}}\left(\widehat{v}^{2}\right)$ and $s_{a_{j}}\left(\widehat{v}^{1}\right)<$ $\left.s_{a_{j}}\left(\widehat{v}^{2}\right)\right)$. This yields a contradiction with Positive Responsiveness as by definition $\widehat{v}^{1} \succ_{\left\{a_{i} a_{j}\right\}} \widehat{v}^{2}$ implying that $a_{j} \notin W\left(\widehat{v}^{2}\right)$.

$2-s_{a_{i}}\left(\widehat{v}^{1}\right) \leq s_{a_{i}}\left(\widehat{v}^{2}\right)$ and $s_{a_{j}}\left(\widehat{v}^{1}\right) \geq s_{a_{j}}\left(\widehat{v}^{2}\right)$. This yields a contradiction with the assumption that $r^{-}<r^{+}$.

$3-s_{a_{i}}\left(\widehat{v}^{1}\right)<s_{a_{i}}\left(\widehat{v}^{2}\right)$ and $s_{a_{j}}\left(\widehat{v}^{1}\right)<s_{a_{j}}\left(\widehat{v}^{2}\right)$. In this case, the vote distribution $\widehat{v}^{2}-\widehat{v}^{1}$ can be defined. Using an argument similar to that used to prove lemma 6 , we can show that $a_{i} \in W\left(v^{2}-v^{1}\right)$. However, one can prove that $s_{a_{i}}\left(\widehat{v}^{2}-\widehat{v}^{1}\right) / s_{a_{j}}\left(\widehat{v}^{2}-\widehat{v}^{1}\right)<$ $r^{-}$which entails a contradiction with the definition of $r^{-}$.

$4-s_{a_{i}}\left(\widehat{v}^{1}\right)>s_{a_{i}}\left(\widehat{v}^{2}\right)$ and $s_{a_{j}}\left(\widehat{v}^{1}\right)>s_{a_{j}}\left(\widehat{v}^{2}\right)$. In this case, the vote distribution $\widehat{v}^{1}-\widehat{v}^{2}$ can be defined. Using an argument similar to that used to prove lemma 6 , we can show that $a_{j} \in W\left(\widehat{v}^{1}-\widehat{v}^{2}\right)$. However, one can prove that $s_{a_{i}}\left(\widehat{v}^{1}-\widehat{v}^{2}\right) / s_{a_{j}}\left(\widehat{v}^{1}-\widehat{v}^{2}\right)>$ $r^{+}$which entails a contradiction with the definition of $r^{+}$.

Lemma 9. Let an aggregation procedure $W: V \rightrightarrows A$ satisfy Universal Domain, Anonymity, Positive Responsiveness, General Abstention and Coalition Permanency. For all $v^{1}, v^{2} \in V \backslash v^{0}$ such that $\frac{s_{a_{i}}\left(v^{1}\right)}{s_{a_{j}}\left(v^{1}\right)} \geq \frac{s_{a_{i}}\left(v^{2}\right)}{s_{a_{j}}\left(v^{2}\right)}$ for any $a_{j} \neq a_{i}$,

$$
a_{i} \in W\left(v^{2}\right) \Longrightarrow a_{i} \in W\left(v^{1}\right)
$$

Proof. Take first any two vote distributions $v^{1}$ and $v^{2}$ with $v^{1}, v^{2} \in V \backslash v^{0}$, and $\frac{s_{a_{i}}\left(v^{1}\right)}{s_{a_{j}}\left(v^{1}\right)}>\frac{s_{a_{i}}\left(v^{2}\right)}{s_{a_{j}}\left(v^{2}\right)}$ for any $a_{j} \neq a_{i}$. Hence, whenever $a_{i} \in W\left(v^{2}\right)$, by Lemma 8, $a_{j} \notin W\left(v^{1}\right)$ so that, as $W\left(v^{1}\right) \neq \emptyset$, we have that $W\left(v^{1}\right)=\left\{a_{i}\right\}$ as desired.

Let us now take any two vote distributions $v^{1}$ and $v^{2}$ with $\frac{s_{a_{i}}\left(v^{1}\right)}{s_{a_{h}}\left(v^{1}\right)}=\frac{s_{a_{i}}\left(v^{2}\right)}{s_{a_{h}}\left(v^{2}\right)}=m$ for some $a_{h} \in A$ and some $m \in \mathbb{Q}_{+}$, and $\frac{s_{a_{i}}\left(v^{1}\right)}{s_{a_{j}}\left(v^{1}\right)}>\frac{s_{a_{i}}\left(v^{2}\right)}{s_{a_{j}}\left(v^{2}\right)}$ for any $a_{j} \neq a_{i}, a_{h}$. W.l.o.g. we assume that $a_{i}=a_{1}$ and $a_{h}=a_{2}$.

Let us assume first that $m=0$. Hence, both $s_{a_{1}}\left(v^{1}\right)$ and $s_{a_{1}}\left(v^{2}\right)$ must be equal to zero, so that for all $a_{j} \neq a_{1}, a_{2}, \frac{s_{a_{1}}\left(v^{2}\right)}{s_{a_{j}}\left(v^{2}\right)}=\frac{s_{a_{1}}\left(v^{1}\right)}{s_{a_{j}}\left(v^{1}\right)}=0$, a contradiction.

Let us now assume that $0<m<\infty$. The vote distribution $v^{1}$ leads, by Lemma 5 , to the same outcome as the vote distribution $u^{1}$ with score vector

$$
s\left(u^{1}\right)=s\left(v^{1}\right) s_{a_{2}}\left(v^{2}\right)=\left(m s_{a_{2}}\left(v^{1}\right) s_{a_{2}}\left(v^{2}\right), s_{a_{2}}\left(v^{1}\right) s_{a_{2}}\left(v^{2}\right), s_{a_{3}}\left(v^{1}\right) s_{a_{2}}\left(v^{2}\right), \ldots, s_{a_{k}}\left(v^{1}\right) s_{a_{2}}\left(v^{2}\right)\right) .
$$


Similarly, the vote distribution $v^{2}$ leads to the same outcome as the vote distribution $u^{2}$ with score vector

$s\left(u^{2}\right)=s\left(v^{2}\right) s_{a_{2}}\left(v^{1}\right)=\left(m s_{a_{2}}\left(v^{2}\right) s_{a_{2}}\left(v^{1}\right), s_{a_{2}}\left(v^{2}\right) s_{a_{2}}\left(v^{1}\right), s_{a_{3}}\left(v^{2}\right) s_{a_{2}}\left(v^{1}\right), \ldots, s_{a_{k}}\left(v^{2}\right) s_{a_{2}}\left(v^{1}\right)\right)$.

By assumption, for all $a_{j} \neq a_{1}, a_{2}, \frac{s_{a_{1}}\left(v^{1}\right)}{s_{a_{j}}\left(v^{1}\right)}>\frac{s_{a_{1}}\left(v^{2}\right)}{s_{a_{j}}\left(v^{2}\right)}$, so that $\frac{s_{a_{1}}\left(u^{1}\right)}{s_{a_{j}}\left(u^{1}\right)}>\frac{s_{a_{1}}\left(u^{2}\right)}{s_{a_{j}}\left(u^{2}\right)}$ for all $a_{j} \neq a_{1}, a_{2}$ which amounts to say

$$
\frac{m s_{a_{2}}\left(v^{1}\right) s_{a_{2}}\left(v^{2}\right)}{s_{a_{j}}\left(v^{1}\right) s_{a_{2}}\left(v^{2}\right)}>\frac{m s_{a_{2}}\left(v^{2}\right) s_{a_{2}}\left(v^{1}\right)}{s_{a_{j}}\left(v^{2}\right) s_{a_{2}}\left(v^{1}\right)} \text { for all } a_{j} \neq a_{1}, a_{2}
$$

which is equivalent to

$$
s_{a_{j}}\left(v^{2}\right) s_{a_{2}}\left(v^{1}\right)>s_{a_{j}}\left(v^{1}\right) s_{a_{2}}\left(v^{2}\right) \text { for all } a_{j} \neq a_{1}, a_{2},
$$

which ultimately implies that $s_{a_{j}}\left(u^{2}\right)>s_{a_{j}}\left(u^{1}\right)$ for all $a_{j} \neq a_{1}, a_{2}$.

Consider finally the vote distribution $u^{3}$ which generates the following score vector:

$$
s_{a_{3}}\left(u^{3}\right)=s_{a_{3}}\left(u^{1}\right)
$$

and

$$
s_{a_{j}}\left(u^{3}\right)=s_{a_{j}}\left(u^{2}\right) \text { for any } a_{j} \neq a_{3} .
$$

This entails that, by construction, $u^{3} \succ_{\left\{a_{1} a_{3}\right\}} u^{2}$ as $s_{a_{1}}\left(u^{3}\right)=s_{a_{1}}\left(u^{2}\right), s_{a_{3}}\left(u^{3}\right)=$ $s_{a_{3}}\left(u^{1}\right)<s_{a_{3}}\left(u^{2}\right)$ and $s_{a_{j}}\left(u^{3}\right)=s_{a_{j}}\left(u^{2}\right)$ for all $a_{j} \neq a_{1}, a_{3}$.

Iterating the process for the $k-3$ remaining alternatives we have $u^{1}=u^{k} \succ_{\left\{a_{1} a_{k}\right\}}$ $u^{k-1} \succ_{\left\{a_{1} a_{k-1}\right\}} \cdots \succ_{\left\{a_{1} a_{5}\right\}} u^{4} \succ_{\left\{a_{1} a_{4}\right\}} u^{3} \succ_{\left\{a_{1} a_{3}\right\}} u^{2}$. Hence, by Positive Responsiveness, whenever $a_{i} \in W\left(u^{2}\right)$, we have that $a_{i} \in W\left(u^{1}\right)$. As by definition $W\left(v^{1}\right)=W\left(u^{1}\right)$ and $W\left(v^{2}\right)=W\left(u^{2}\right)$, we can write that whenever $a_{i} \in W\left(v^{1}\right)$, we have that $a_{i} \in W\left(v^{2}\right)$, as desired.

The case in which $m=\infty$ remains to be considered. However, the case is quite similar to the previous one with the feature that both $s_{a_{2}}\left(v^{1}\right)$ and $s_{a_{2}}\left(v^{2}\right)$ must be equal to zero. Hence the score vectors of $u^{1}$ and $u^{2}$ satisfy $s\left(u^{1}\right)=s_{a_{1}}\left(v^{2}\right) s\left(v^{1}\right)$ and $s\left(u^{2}\right)=s_{a_{1}}\left(v^{1}\right) s\left(v^{2}\right)$.

In order to finish the proof, one has to consider the cases in which, for some two vote distributions $v^{1}$ and $v^{2}$ with $\frac{s_{a_{i}}\left(v^{2}\right)}{s_{a_{h}}\left(v^{2}\right)}=\frac{s_{a_{1}}\left(v^{1}\right)}{s_{a_{h}}\left(v^{1}\right)}=m_{h}$ for several alternatives $a_{h}$. However, the logic in the proofs remains true almost verbatim.

In what follows we show that the proof of theorem 2 is built on the previous lemmata.

1- If $v=0$ then, by General Abstention, $W(v)=A$. 
2- For all $v \in V$, let $r_{i h} \in \mathbb{Q}_{+}$denote the ratio such that $s_{a_{i}}(v)=r_{i h} s_{a_{h}}(v)$ for all $a_{h} \in A$. By lemmata 5 and 7 , there exists one and only one $r_{i j} \in \mathbb{Q}_{+}, 0<r_{i j}<\infty$, such that $s_{a_{i}}(v)=r_{i j} s_{a_{j}}(v)$ for all $a_{i}, a_{j} \in A$ such that both $a_{i}, a_{j} \in W(v)$ for some $v \in V \backslash v^{0}$.

Given that $a_{i} \in W(v)$, Lemma 9 implies that, for all $v^{\prime} \in V$ with $s_{a_{i}}\left(v^{\prime}\right) \geq$ $r_{i h} s_{a_{h}}\left(v^{\prime}\right)$ for all $a_{h} \neq a_{i}, a_{i} \in W\left(v^{\prime}\right)$. Moreover, by the uniqueness of $r_{a_{i} a_{j}}, a_{j} \notin$ $W\left(v^{\prime}\right)$ whenever $s_{a_{i}}\left(v^{\prime}\right)>r_{i j} s_{a_{j}}\left(v^{\prime}\right)$. Similarly, for all $v^{\prime}$ with $s_{a_{i}}\left(v^{\prime}\right)<r_{i j} s_{a_{j}}\left(v^{\prime}\right)$. Lemma 8 implies that, as $a_{j} \in W(v), a_{i} \notin W\left(v^{\prime}\right)$. Notice that this reasoning also determines the family of vote distributions at which any subset of alternatives are in the winning set.

3- Consider all $a_{i}, a_{j} \in A$ such that there does not exist any $v \in V$ in which they both belong to the winning set, i.e. no $v$ for which $a_{i}, a_{j} \in W(v)$. Let then

$$
\hat{R}^{i}=\left\{r \in \mathbb{Q}_{+} \mid \exists v \in V \text { s.t. } \frac{s_{a_{i}}(v)}{s_{a_{j}}(v)}=r \text { and } a_{i} \in W(v)\right\}
$$

and

$$
\hat{R}^{j}=\left\{r \in \mathbb{Q}_{+} \mid \exists v \in V \text { s.t. } \frac{s_{a_{i}}(v)}{s_{a_{j}}(v)}=r \text { and } a_{j} \in W(v)\right\}
$$

Notice that, by assumption, $\hat{R}^{j} \cap \hat{R}^{i}=\emptyset$. Moreover, by lemma 3 both $\hat{R}^{j}$ and $\hat{R}^{i}$ are not empty and, since $N$ is finite, they are also finite. Let $r^{j}=\max \hat{R}^{j}$ and $r^{i}=\min \hat{R}^{i}$. Again by Lemma $3 r^{j}<\infty$ and $r^{i}>0$.

Assume by contradiction that $r^{j} \geq r^{i}$. By lemma $5, r^{j} \neq r^{i}$ since, $\hat{R}^{j} \cap \hat{R}^{i}=\emptyset$. So we are left with the case $r^{j}>r^{i}$. Let $\widehat{v}^{1}$ and $\widehat{v}^{2} \in V$ be such that $\frac{s_{a_{i}}\left(\widehat{v}^{1}\right)}{s_{a_{j}}\left(\widehat{v}^{1}\right)}=r^{j}$ and $a_{j} \in W\left(\widehat{v}^{1}\right)_{j}$ whilst $a_{i} \notin W\left(\widehat{v}^{1}\right)$ and $\frac{s_{a_{i}}\left(\widehat{v}^{2}\right)}{s_{a_{j}}\left(\widehat{v}^{2}\right)}=r^{i}$ and $a_{i} \in W\left(\widehat{v}^{2}\right)$ whilst $a_{j} \notin W\left(\widehat{v}^{2}\right)$. By assumption we have $\frac{s_{a_{i}}\left(\widehat{v}^{1}\right)}{s_{a_{j}}\left(\widehat{v}^{1}\right)}>\frac{s_{a_{i}}\left(\widehat{v}^{2}\right)}{s_{a_{j}}\left(\widehat{v}^{2}\right)}$ which entails, by lemma 8 that $a_{j} \notin W\left(\widehat{v}^{1}\right)$, the desired contradiction.

Let us set $r \in \mathbb{R}_{+}$such that $r^{j}<r<r^{i}$. Let us take any $v^{\prime} \in V$ such that either $a_{i} \in W\left(v^{\prime}\right)$ or $a_{j} \in W\left(v^{\prime}\right)$ so that $\frac{s_{a_{i}}\left(v^{\prime}\right)}{s_{a_{j}}\left(v^{\prime}\right)} \in \hat{R}^{j} \cup \hat{R}^{i}$. If moreover $\frac{s_{a_{i}}\left(v^{\prime}\right)}{s_{a_{j}}\left(v^{\prime}\right)}>r$ then, by definition $\frac{s_{a_{i}}\left(v^{\prime}\right)}{s_{a_{j}}\left(v^{\prime}\right)}>r^{j}$ so that necessarily $a_{i} \in W\left(v^{\prime}\right)$. If, conversely, $\frac{s_{a_{i}}\left(v^{\prime}\right)}{s_{a_{j}}\left(v^{\prime}\right)}<r<r^{i}$ so that $a_{j} \in W\left(v^{\prime}\right)$.

\section{References}

[1] J. Apesteguia, M.A. Ballester, and R. Ferrer. On the Justice of Decision Rules. Review of Economic Studies, 71:1-16, 2011.

[2] K. Arrow. Social Choice and Individual Values. New Haven and London. Yale University Press, 1951. 
[3] G. Asan and R. Sanver. Another Characterization of the Majority Rule. Economic Letters, 75:409-413, 2002.

[4] G. Asan and R. Sanver. Maskin Monotonic Aggregation Rules. Economic Letters, 91:179-183, 2006.

[5] D. Austen-Smith and J. Banks. Positive Political Theory I: Collective Preference. Ann Arbor:University of Michigan Press., 1999.

[6] D.E. Campbell. A Characterization of Simple Majority Rule for Restricted Domains. Economic Letters, 28:307-310, 1988.

[7] D.E. Campbell and J.S. Kelly. A Simple Characterization of Majority Rule. Economic Theory, 15:689-700, 2000.

[8] E. Cantillon and A. Rangel. A Graphical Analysis of some Basic Results in Social Choice. Social Choice and Welfare, 19:587-611, 2002.

[9] P.Y. Chebotarev and E. Shamis. Characterizations of Scoring Methods for Preference Aggregation. Annals of Operations Research, 80:299-332, 1998.

[10] C. d'Aspremont and C. Gevers. Equity and the Informational Basis of Collective Choice. Review of economic Studies, 44:199-299, 1977.

[11] K. Dowding and M. van Hees. In Praise of Manipulation. British Journal of Political Science, 38:1-15, 2008.

[12] P. C. Fishburn. Condorcet Social Choice Functions. SIAM Journal on Applied Mathematics, 33:469-489, 1977.

[13] M. Fleurbaey. On the Informational Basis of Social Choice. Social Choice and Welfare, 21:347-384, 1993.

[14] A. Gibbard. Manipulation of Voting Schemes : A General Result. Econometrica, 41:587-601, 1973.

[15] I. Gilboa and D. Schmeidler. A Theory of Case-Based Decisions. Cambridge University Press, 2001.

[16] I. Gilboa and D. Schmeidler. Inductive Inference: An Axiomatic Approach. Econometrica, 71:1-26, 2003.

[17] R.E. Goodin and C. List. A Conditional Defense of Plurality Rule: Generalizing May's Theorem in a Restricted Informational Environment. American Journal of Political Science, 50:940-949, 2006. 
[18] R.E. Goodin and C. List. Special Majorities Rationalized. British Journal of Political Science, 36:213-241, 2006.

[19] N. Houy. A Characterization for Qualified Majority Voting Rules. Mathematical Social Sciences, 54:17-24, 2007.

[20] J.-F. Laslier. Tournament Solutions and Majority Voting. Springer-Verlag Berlin Heidelberg New York, 1997.

[21] E. Maskin. Majority Rule, Social Welfare Functions, and Game Forms. In K. Basu, P.K. Pattanaik Pattanaik, and K. Suzumura, editors, Choice, Welfare and Development, Festschrift for Amartya Sen. Clarendon Press, Oxford, 1995.

[22] J. Massó and M. Vorsatz. Weighted Approval Voting. Economic Theory, $36: 129-146,2008$.

[23] K. May. A Set of Independent Necessary and Sufficient Conditions for Simple Majority Decision. Econometrica, 20:680-684, 1952.

[24] N. McCarty and A. Meirowitz. Political Game Theory. Cambridge University Press, 2006.

[25] S. Merill III and J. Nagel. The Effect of Approval Balloting on Strategic Voting Under Alternative Decision Rules. American Political Science Review, 81:509$524,1987$.

[26] R. Myerson. Axiomatic Derivation of Scoring Rules without the Ordering Assumption. Social Choice and Welfare, 12:59-74, 1995.

[27] R. Myerson and R.J. Weber. A Theory of Voting Equilibria. American Political Science Review, 87:102-114, 1993.

[28] T. Palfrey. A Mathematical Proof of Duverger's Law. In P.C. Ordeshook, editor, Models of Strategic Choice in Politics. The University of Michigan Press, 1989.

[29] M. Pivato. Variable-Population Voting Rules. mimeo, 2011.

[30] M. Satterthwaite. Strategy-proofness and Arrow's Conditions: Existence and Correspondence Theorems for Voting Procedures and Social Welfare Functions. Journal of Economic Theory, 10:187-217, 1975.

[31] A.J. Schmidt and N. Babchuk. The Unbrotherly Brotherhood: Discrimination in Fraternal Orders. Phylon, 34:275-282, 1973.

[32] A.K. Sen. Collective Choice and Social Welfare. Holden Day. San Francisco., 1970. 
[33] J. Smith. Aggregation of Preferences with a Variable Electorate. Econometrica, 41:1027-1041, 1973.

[34] G. Woeginger. A New Characterization of the Majority Rule. Economic Letters, 81:89-94, 2003.

[35] P. Young. Social Choice Scoring Functions. SIAM Journal of Applied Mathematics, 27:824-838, 1975. 